\title{
Pontine Hemorrhage Causing Bilateral Hypertrophic Olivary Degeneration
}

Sir, Hypertrophic olivary degeneration (HOD) is a deafferentation syndrome that occurs after a loss of synaptic input from the afferent fibers. HOD occurs in lesions involving the dentato-rubro-olivary system. An unusual finding in this form of transneuronal degeneration is enlargement rather than atrophy of the affected structure. It may occur secondary to pontine lesions, hemorrhage, cavernoma, demyelination, infection, infarction, or postsurgery. On magnetic resonance imaging (MRI), HOD appears as enlargement of the inferior olivary nucleus with increased signal on T2-weighted image (T2-WI). Knowledge of the condition and its characteristic MRI findings can prevent erroneous diagnoses of more serious pathology.

A 52-year-old male hypertensive patient presented with acute onset right-sided hemiparesis and horizontal diplopia on the left gaze for 8 months. There was no headache or vomiting. On examination, he had signs of the left $6^{\text {th }}$ nerve palsy and palatal myoclonus. No history of headache or vomiting or other cranial nerve palsy. There was no intention tremor or any other involuntary movement except for palatal myoclonus. His blood pressure was $140 / 90 \mathrm{~mm} \mathrm{Hg}$ (with medication, amlodipine $10 \mathrm{mg}$ ) at the time of examination. Pulse rate was $80 / \mathrm{min}$, regular. MRI of the head was advised.

MRI was done in a $3 \mathrm{~T}$ scanner (Signa, GE, Wisconsin, USA). T2-WI revealed small hypointense lesion with central hyperintense focus in dorsal pons close to bilateral superior cerebellar peduncle. The lesion was showing blooming on susceptibility weighted angiography (SWAN) sequence. No complete T2 hypointense rim noted to suggest cavernoma. No significant enhancement noted in postcontrast sequence [Figure 1]. T2 and fluid-attenuated inversion recovery hyperintensity also noted in the region of both inferior olivary nucleus. There was mild expansion of the olivary nuclei as well. A diagnosis of old pontine bleed with hypertrophic inferior olivary degeneration was made based on imaging findings.

HOD is a rare form of neuronal degeneration that occurs secondary to any injury that disrupts the afferent fibers to the inferior olive within the dentato-rubro-olivary tract (Triangle of Guillain-Mollaret). It may occur secondary to pontine hemorrhage, cavernoma, demyelination, infection, infarction, or postsurgery. The triangle of Guillain and Mollaret is formed between the red nucleus, ipsilateral inferior olivary nucleus, and contralateral dentate nucleus. Efferent fibers from the dentate nucleus (cerebellorubral and dentatorubral tracts) traverse through the superior cerebellar peduncle and connect to the contralateral red nucleus after decussating in the brachium conjunctivum. The red nucleus sends afferent fibers to ipsilateral inferior olivary nucleus through the central tegmental tract. Olivary nucleus also receives efferent fibers from contralateral dentate nucleus through the inferior cerebellar peduncle, thus forming a triangle. ${ }^{[1]}$

HOD is the end result of transynaptic degeneration of the inferior olivary nucleus after an injury to the dentato-rubro-olivary tract. Many of the nerve fibers from dentate nucleus to inferior olive are primarily inhibitory 


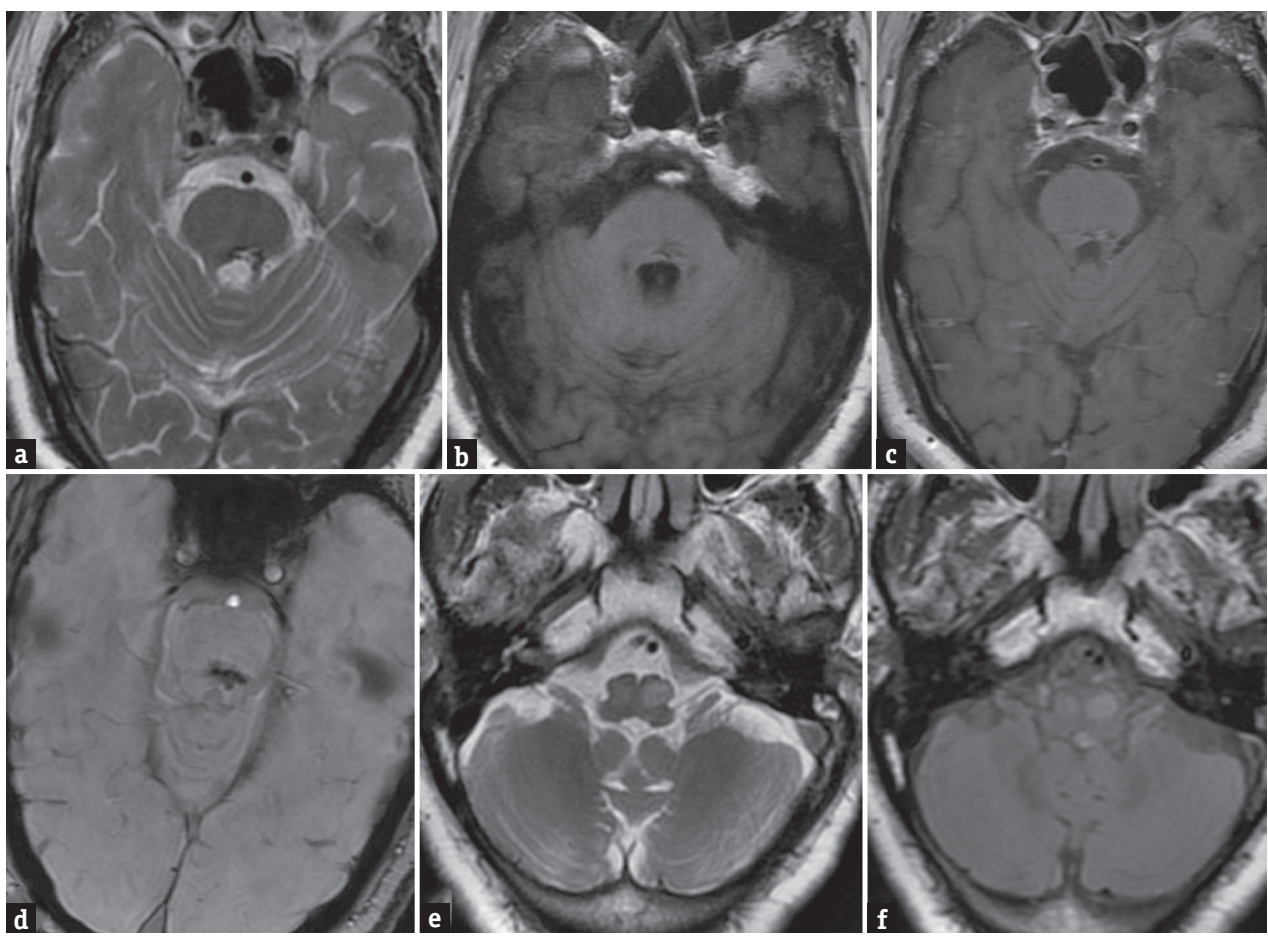

Figure 1: Axial T2-weighted magnetic resonance imaging of the brain (a) shows heterogeneous hypointense lesion in the dorsal pons with small central hyperintensity. The lesion is iso to slightly hypointense on the T1-weighted image (b) with no significant enhancement on post contrast T1-weighted image (c). Lesion shows blooming on SWAN (d). Axial T2-weighted image and fluid attenuated inversion recovery (e and f) at the level of medulla show hyperintensity and mild expansion of bilateral olivary nuclei suggesting bilateral hypertrophic olivary degeneration

or GABArgic. ${ }^{[2]}$ Hence, damage to the dentato-rubral pathway results in loss of inhibitory control with consequent hyperactivity of the olivary neurons leading to abnormal involuntary movements such as palatal myoclonus, choreodystonia, ataxia, and dysarthria. Palatal myoclonus is not always seen, however, if present is almost pathognomonic of this condition. ${ }^{[2,3]}$

Lesions disrupting the superior cerebellar peduncle, brachium conjunctivum, or central tegmental tracts cause deafferentiation of the inferior olivary nucleus and lead to HOD. Lesions in the red nucleus or central tegmental tract cause ipsilateral HOD and that in superior cerebellar peduncle or dentate nucleus causes contralateral HOD. Bilateral HOD occurs when lesion is located in brachium conjunctivum or involves bilateral central tegmental tract or superior cerebellar peduncle. Bilateral HOD is not common. In our case, the lesion was located in the dorsal pons in the midline as well as on the left side, thus probably disrupting the decussating fibers and left dentato-rubral tract causing bilateral HOD ${ }^{[2,4,5]}$

The initial MR change of signal hyperintensity may relate to the initial phases of neuronal hypertrophy, in which gliosis and increased water content are associated with demyelination and vacuolization. The delayed development of hypertrophy is most likely the result of neuronal and astrocytic hypertrophic precursors to cell death, ultimately leading to atrophy ${ }^{[6]}$
On MRI, HOD is characterized by a nonenhancing T1 isointense, T2 hyperintense lesion confined to the olivary nucleus. The signal alteration and swelling of olivary nucleus noted in MRI follows a specific sequence with time. The three stages have been described based on temporal evolution of HOD in MRI. In the first stage, increased signal is noted in the inferior olivary nucleus on T2-WI without hypertrophy and occurs within the first 6 months of ictus. The second stage shows both increased signal and hypertrophy, usually occur after 6 months. The hypertrophy resolves after a period of approximately 3-4 years. The third stage starts when the hypertrophy resolves, however, T2 hyperintensity persists indefinitely. ${ }^{[7]}$

Differential diagnosis includes infarction, demyelinating disease, malignancy - such as primary brainstem astrocytoma or metastasis, infections, and inflammation. The absence of enhancement helps distinguish HOD from tumor, inflammation, or infection. Localization of the altered signal to the inferior olivary nucleus, lack of enhancement, timing of signal changes and swelling, and importantly inciting lesion located in the dentato-rubro cerebellar tract clinches the diagnosis.

We found T2 hypointense lesion with central hyperintense focus in the dorsal pons predominantly in the left side and midline as well. The peripheral hypointensity could be due to hemosiderin and central 
hyperintensity due to methemoglobin stage of bleed. It is not clear whether it was attributed to hypertensive bleed or hemorrhage from occult cavernous angioma. No prior imaging was available in our patient.

The treatment is according to the symptoms. Palatal myoclonus is difficult to treat, however, successful management of symptoms with benzodiazepines and carbamazepine has been reported.

HOD is caused by lesions in the dentatorubral or central tegmental tracts. Familiarity with the characteristic imaging findings of this rare phenomenon is necessary to avoid misdiagnosis and prevent unnecessary intervention.

\section{Financial support and sponsorship}

Nil.

\section{Conflicts of interest}

There are no conflicts of interest.

Suprava Naik, Rajendra V. Phadke', Vivek Agarwal', Vivek Singh ${ }^{1}$, Sanjeev Kumar Bhoi ${ }^{2}$

Departments of Radiodiagnosis and ${ }^{2}$ Neurology, All India Institute of Medical Science, Bhubaneswar, Odisha, ${ }^{1}$ Department of Radiodiagnosis, Sanjay Gandhi Postgraduate Institute of Medical Science, Lucknow, Uttar Pradesh, India

Address for correspondence: Dr. Suprava Naik, Department of Radiodiagnosis, All India Institute of Medical Sciences, Bhubaneswar, Odisha, India. E-mail: drsuprava.rd@gmail.com

\section{REFERENCES}

1. Guillain G, Mollaret P. Deux de myoclonies synchrones et rhythmees velopharyngolaryngo-oculo-diaphragmatiques. Rev Neurol 1931;2:545-66.
2. Akar S, Drappatz J, Hsu L, Blinder RA, Black PM, Kesari S. Hypertrophic olivary degeneration after resection of a cerebellar tumor. J Neurooncol 2008;87:341-5.

3. Lapresle J. Rhythmic palatal myoclonus and the dentato-olivary pathway. J Neurol 1979;220:223-30.

4. Macht S, Hänggi D, Turowski B. Hypertrophic olivary degeneration following pontine cavernoma hemorrhage: A typical change accompanying lesions in the Guillain-Mollaret triangle. Clin Neuroradiol 2009;19:235-7.

5. Gerace C, Fele MR, Luna R, Piazza G. Neurological picture. Bilateral hypertrophic olivary degeneration. J Neurol Neurosurg Psychiatry 2006;77:73.

6. Goto N, Kakimi S, Kaneko M. Olivary enlargement: Stage of initial astrocytic changes. Clin Neuropathol 1988;7:39-43.

7. Goyal M, Versnick E, Tuite P, Cyr JS, Kucharczyk W, Montanera W, et al. Hypertrophic olivary degeneration: Metaanalysis of the temporal evolution of MR findings. AJNR Am J Neuroradiol 2000;21:1073-7.

This is an open access article distributed under the terms of the Creative Commons Attribution-NonCommercial-ShareAlike 3.0 License, which allows others to remix, tweak, and build upon the work non-commercially, as long as the author is credited and the new creations are licensed under the identical terms.

\begin{tabular}{|l|l|}
\hline \multicolumn{2}{|c|}{ Access this article online } \\
\hline Quick Response Code: & Website: \\
\hline
\end{tabular}

How to cite this article: Naik S, Phadke RV, Agarwal V, Singh V, Bhoi SK. Pontine hemorrhage causing bilateral hypertrophic olivary degeneration. J Neurosci Rural Pract 2017;8:498-500.

(C) 2017 Journal of Neurosciences in Rural Practice | Published by Wolters Kluwer - Medknow 